\title{
A blood RNA transcript signature for TB exposure in household contacts
}

\author{
Philip Kam Weng Kwan ${ }^{1 \dagger}$, Balamurugan Periaswamy ${ }^{2 \dagger}$, Paola Florez De Sessions², Wenwei Lin ${ }^{1}$, James S. Molton,
} Claire M. Naftalin', Ahmad Nazri Mohamed Naim², Martin L. Hibberd ${ }^{2,3,4}$ and Nicholas I. Paton 1,3*(i)

\begin{abstract}
Background: Current tools for diagnosing latent TB infection (LTBI) detect immunological memory of past exposure but are unable to determine whether exposure is recent. We sought to identify a whole-blood transcriptome signature of recent TB exposure.
\end{abstract}

Methods: We studied household contacts of TB patients; healthy volunteers without recent history of TB exposure; and patients with active TB. We performed whole-blood RNA sequencing (in all), an interferon gamma release assay (IGRA; in contacts and healthy controls) and PET/MRI lung scans (in contacts only). We evaluated differentiallyexpressed genes in household contacts (log2 fold change $\geq 1$ versus healthy controls; false-discovery rate $<0.05$ ); compared these to differentially-expressed genes seen in the active TB group; and assessed the association of a composite gene expression score to independent exposure/treatment/immunological variables.

Results: There were 186 differentially-expressed genes in household contacts ( $n=26$, age 22-66, 46\% male) compared with healthy controls ( $n=5$, age 29-38, 100\% male). Of these genes, 141 (76\%) were also differentially expressed in active TB ( $n=14$, age $27-69,71 \%$ male). The exposure signature included genes from inflammatory response, type I interferon signalling and neutrophil-mediated immunity pathways; and genes such as BATF2 and SCARF1 known to be associated with incipient TB. The composite gene-expression score was higher in IGRA-positive contacts $(P=0.04)$ but not related to time from exposure, isoniazid prophylaxis, or abnormalities on PET/MRI (all $P>0.19$ ).

Conclusions: Transcriptomics can detect TB exposure and, with further development, may be an approach of value for epidemiological research and targeting public health interventions.

Keywords: Tuberculosis; gene expression, Biomarkers, TB exposure, TB infection; RNA sequencing, Whole blood

\section{Background}

Following exposure to and infection with tuberculosis (TB) many individuals enter a state of latent TB infection (LTBI) $[1,2]$. An estimated $25 \%$ of the world's population has LTBI, with the highest prevalence found in the WHO

\footnotetext{
* Correspondence: nick_paton@nuhs.edu.sg

†Philip Kam Weng Kwan and Balamurugan Periaswamy contributed equally to this work.

'Department of Medicine, Yong Loo Lin School of Medicine, National University of Singapore, NUHS Tower Block Level 10, 1E Kent Ridge Road, Singapore 119228, Singapore

${ }^{3}$ London School of Hygiene \& Tropical Medicine, London, UK

Full list of author information is available at the end of the article
}

Southeast Asia (31\%) and Western Pacific regions (28\% overall, $13 \%$ in Singapore), compared to $11-22 \%$ in the other regions of the world [3-5]. LTBI forms a large reservoir from which new active TB cases develop. Prevention of transmission and consequent replenishment of the LTBI reservoir and treatment of LTBI to prevent development of active disease are essential if the goals of the WHO's End TB are to be achieved [6].

Current tools for diagnosis of LTBI that depend on detection of an immune response to exogenously-administered TB antigens - in vivo as the tuberculin skin test (TST), or ex vivo as an interferon gamma release assay (IGRA) [7] -

(c) The Author(s). 2020 Open Access This article is licensed under a Creative Commons Attribution 4.0 International License, which permits use, sharing, adaptation, distribution and reproduction in any medium or format, as long as you give appropriate credit to the original author(s) and the source, provide a link to the Creative Commons licence, and indicate if changes were made. The images or other third party material in this article are included in the article's Creative Commons licence, unless indicated otherwise in a credit line to the material. If material is not included in the article's Creative Commons licence and your intended use is not permitted by statutory regulation or exceeds the permitted use, you will need to obtain permission directly from the copyright holder. To view a copy of this licence, visit http://creativecommons.org/licenses/by/4.0/ The Creative Commons Public Domain Dedication waiver (http://creativecommons.org/publicdomain/zero/1.0/) applies to the data made available in this article, unless otherwise stated in a credit line to the data. 
detect immunological memory of past exposure but are unable to determine whether exposure is recent. A test that could identify recent exposure may be of value for epidemiological research as well as for public health, identifying areas with ongoing high rates of transmission where active case-finding and infection control measures could be enhanced to prevent new cases of LTBI. More than half of prevalent culture positive TB disease is asymptomatic, and much transmission goes unrecognised $[8,9]$.

Transcriptome-based signatures have been shown to detect incipient disease in individuals with LTBI that precedes the onset of clinical disease, indicating the potential of transcriptomics to respond dynamically to subclinical events in the TB disease spectrum [10-12]. PET-based imaging studies have demonstrated the existence of early changes, mainly in lymph nodes, following exposure to TB, $[13,14]$ and this suggests that there may be sufficient disease activity early after exposure to drive a transcriptome response. In this study, we aimed to determine whether a transcriptome signature can be identified that differentiates individuals with recent $\mathrm{TB}$ exposure from those without; to explore the biological basis of the early exposure signature; and to examine the relationship between the signature and relevant clinical and demographic factors.

\section{Methods}

\section{Participants and sample collection}

We performed a cross-sectional study, comparing gene expression patterns in three participant groups: a group with recent TB exposure, without active clinical TB (household contact group); group without recent $\mathrm{TB}$ exposure or active clinical TB (healthy control group, as a reference to determine differential gene expression in the TB exposed); and a group of patients with clinical TB (active TB group; to provide context for the TB exposure signature).

The household contact group comprised 26 adult contacts of smear-positive index TB cases. They had participated in a PET-based imaging study conducted at the National University Hospital (NUH), Singapore from 2013 to 2015, for which the main eligibility criteria were age over 21 years; residing in the same house as a newlydiagnosed smear-positive pulmonary TB patient for at least 1 month prior to start of anti-TB treatment in the index case [14-16]; receiving no previous treatment for active TB; having no evidence of active clinical TB; and no severe uncontrolled diabetes (Supplementary Table 1). Demographic, clinical and exposure data were collected. Time from exposure was estimated as the difference between the date that the index patient first reported symptoms or began to live in the same household as the contact, whichever was later, and the date that the blood was drawn for transcriptome profiling. An IGRA test was performed (QuantiFERON-TB Gold In-Tube Test, Qiagen, Hilden, Germany) and repeated after approximately 3 months if the initial test was negative or indeterminate. A whole blood sample was drawn into a Tempus RNA preservation tube (Thermo Fisher Scientific, Massachusetts, USA), frozen within $2 \mathrm{~h}$ of collection, and stored at $-80^{\circ} \mathrm{C}$ until analysis. A PET/MRI scan was performed, as previously described [14].

The healthy control group comprised 5 volunteers (recruited from a database) who had previously participated in a PK/PD study at NUH from 2015 to 2016. Participants with immune deficiency, past history of $\mathrm{TB}$, or known contact with a TB index case in the previous year were not enrolled (Supplementary Table 1). Participants were assessed clinically (symptom questionnaire) to rule out active TB; demographic and clinical data were collected; an IGRA test was performed; and whole blood collected in an RNA preservation tube (as above).

The active TB group comprised 14 patients who had a clinical diagnosis of pulmonary TB (presence of symptoms, chest X-ray findings and positive GeneXpert; with later microbiological confirmation); and less than 1 week of standard combination treatment (Supplementary Table 1). They had participated in a PET-based imaging study at NUH from 2013 to 2015. No IGRA testing was performed. A whole blood sample was collected in an RNA preservation tube and stored (as above).

All study protocols were approved by the Singapore National Healthcare Group Domain Specific Review Board (NHG DSRB) and all participants gave written informed consent.

\section{Extraction of total RNA from peripheral blood and RNA sequencing}

Samples were thawed, total RNA extracted, and DNAsetreatment was performed using the column-based Tempus $^{\mathrm{TM}}$ Spin RNA Isolation kit (ThermoFisher Scientific, Massachusetts, USA). RNA was quantified using the Agilent 2100 Bioanalyzer (Agilent Technologies, California, USA). cDNA libraries were constructed using the TruSeq total RNA V2 (Illumina, USA). Total RNA was reverse transcribed into cDNA and ligated with RNA sequencing adaptors. RNA sequencing was performed on Illumina HiSeq Rapid v2 (2x76bp) at the Genome Institute of Singapore (GIS).

\section{RNASeq data analyses and functional annotation}

Sequenced reads (paired-end FASTQ files) were mapped to the Genome Reference Consortium Human Build 38 release 86 (GRCh38.r86) by using STAR aligner [17]. The aligned reads were counted for each gene using HTSeq [18]. Sample read counts were adjusted for library size and normalized using Trimmed Mean of Mvalues (TMM) method and multidimensional scaling plots were used to detect any outlier samples (none found) using Bioconductor package EdgeR [19]. 
Differential gene expression was assessed using the exact test in EdgeR. Genes were considered differentially expressed if they had a false-discovery rate (FDR, Benjamini-Hochberg) [20] of less than 0.05 and at least a $\log 2$ fold change of \pm 1 .

Gene expression in the household contact group was compared with the healthy control group to identify differentially expressed genes (the "TB exposure signature"). The nature of the TB exposure signature was explored further by creating a comprehensive protein network representation of the proteins associated with the genes in the signature using Search Tool for the Retrieval of Interacting Genes/Proteins (STRING) [21]. Analysis of the associated functional pathways was performed using the GeneOntology (GO) Biological Processes on STRING.

The probability of identifying overlap genes in the TB exposure signature with genes overexpressed in active TB (compared with the same control group; using the same FDR thresholds) was tested by exact hypergeometric probability method [22]. The association between the relative magnitudes of overexpression of genes in the two groups was assessed by Pearson's correlation and the difference by Wilcoxon signed-rank test.

An exposure risk score to quantify the strength of the TB exposure signature was calculated for each individual in the household contact group using the normalized gene expression values of the genes in the TB exposure signature, following an approach described previously [23-25]. Within the household contact group, the relationship between the exposure risk score and exposure variables - time from first exposure (at least 60 days versus less than 60 days), use of isoniazid prophylaxis at the time of the blood draw, IGRA positivity, and presence of abnormalities on PET/MRI scan - was assessed by Mann-Whitney U test.

The area under the curve (AUC) of the receiver operating characteristic (ROC) curve obtained with the exposure risk score from the TB-exposed household contacts and TB unexposed healthy control groups was calculated using a parametric method and the optimal cut-off for distinguishing between those with and without exposure was determined (ROCR package) [26].

All analyses and figures were generated using the $R$ software or custom Python scripts.

The sample size for household contacts was determined by pragmatic considerations of the availability of wholeblood RNA-preserved samples from previous household contact studies using PET-MRI performed in our group, assay costs and the exploratory nature of the study that did not intend to validate the signature against diseaseprogression outcomes; the sample size for the healthy control group and active TB group were based on similar pragmatic considerations and experience with other studies where comparison groups of this size typically allow detection of differential gene expression in a study group of interest.

\section{Results}

The 26 participants in the household contact group (46\% male, mean age 42 years, $46 \%$ Chinese ethnicity) lived in 16 discrete households (ten households contributed one contact; three households, two contacts; two households, three contacts; one household, four contacts). They were studied at a median of 95 (range 0-752) days from estimated first exposure to infection. Thirteen (50\%) were IGRA positive and 4 (15\%) had started isoniazid treatment a median of 17 days (range 7-44 days) prior to the study day; 7 had abnormalities (mainly lymphadenopathy) observed on PET/MRI scan, as previously reported [14]. Participants were otherwise well, without any significant underlying medical conditions.

Of the 5 participants in the control group (100\% male, mean age 29 years, $100 \%$ Chinese ethnicity), two were IGRA positive (no recent exposure history). Participants did not have any significant underlying medical conditions.

The 14 participants in the active $T B$ group (71\% male, mean age 51 years, $40 \%$ Chinese ethnicity) all had drugsusceptible TB and had been on treatment for a median of 5 days (range 2-21 days) on the day of the study. Seven (50\%) were smear positive; 7 (50\%) had cavitation on the chest-X-ray; 5 (36\%) still had fever and 8 (57\%) cough at the time of the study. Six of the patients had diabetes mellitus.

We found 186 genes that were differentially expressed (180 induced, 6 repressed) in household contacts compared to healthy controls (listed in Supplementary Table 2). Of these 186 genes, 141 (76\%) were also differentially expressed in the patients with active TB (versus healthy controls); there was a moderate association between the relative magnitude of expression of individual genes in the two groups $(\mathrm{r}=0.66, P<0.0001)$ although overall expression values were lower in the household contacts $(P<0.00001$; Supplementary Figure 1$)$.

Of these 186 genes, 69 have been reported previously in published human TB-related signatures (Supplementary Table 3), including 68 previously reported for active TB (including CD274, IRF7, IFI6) [23-25, 27-35]; 5 in a 126-gene signature of LTBI (ADM, DUSP2, IER3, OSM, SOCS3; $P<0.011$ for overlap) [33]; 4 in a 16-gene signature of incipient TB in those with LTBI (ANKRD22, BATF2, SERPING1, SCARF1; $P<0.001$ for overlap), [10] and 2 in a 3-gene signature of incipient TB in TB contacts (BATF2 and SCARF1; $P<0.0001)$ [36].

Analysis of the proteins associated with all 186 genes and functional analysis of protein pathways revealed clusters of proteins from multiple immune response pathways including the inflammatory response, type I 
interferon signalling and neutrophil-mediated immunity pathways (Fig. 1, Supplementary Table 4).

The TB exposure risk score, a composite score calculated from the values of expression of the 186 genes, did not differ by time from first exposure (median 17,758 $[11,543-31,650]$ versus $27,005[10,618-62,190]$ in those with exposure onset $\leq 60$ days versus $>60$ days prior to sampling respectively, $P=0.29$ ); by use of isoniazid prophylaxis (median 38,432 [16,518 - 62,190] versus 22, 758 [10,618 to 44,448$]$ in those taking versus not taking isoniazid prophylaxis respectively; $P=0.197$ ); or by presence of lung abnormalities on PET/MRI scan (median 21,400 [15,836 to 43,919 ] versus $26,510[10,618$ to 62 , $190]$ in those with PET/MRI abnormalities versus no abnormalities respectively; $P=0.955$ ).

However, the risk score was higher in contacts who were IGRA positive (median 28,028 [15,836 - 62,190]) versus IGRA negative $(17,469(10,618-44,448] ; P=$ 0.044 ). The risk score was positive (above the threshold identified on ROC analysis) in 23 of 26 (88\%) contacts, of whom only 13 (56\%) were IGRA positive.

\section{Discussion}

We identified a 186-gene signature that can differentiate people with recent exposure to TB from those without recent exposure. Transcriptomic signatures have been shown previously to differentiate active TB from LTBI, $[23,25,28,29,32,33,35,37]$ from other infections, [23, $24,27,38]$ and from healthy individuals $[25,27,28,30$, $32,33]$; to differentiate LTBI from those who are uninfected [33, 34, 39]; to identify those at risk of relapse after treatment for TB disease [40]; and to identify those with LTBI (including household contacts) who are at risk of reactivation $[10,11,25,36]$. However, this is the first time transcriptomics has been used to differentiate those with and without recent TB exposure. Our study adds to the potential applications for transcriptomicsbased testing in an area of special need given the limitations of current diagnostic tools in early TB disease.

This signature is biologically plausible as a signature of TB exposure. The majority of genes were also found in our patients with active TB as well as many published active-TB signatures. Several genes are highly specific for TB (i.e. CD274, IRF7, IFI6) [27] and others have been found in signatures indicative of incipient/subclinical TB (such as BATF2, SCARF1 and SERPING1) [10, 36]. Many of the genes in the signature are non-specific inflammatory markers and overexpression might reflect recent exposure to respiratory or other circulating infections such as dengue fever that are common in Singapore. However, this is unlikely to explain our findings because of the strong epidemiological evidence for recent exposure to a case of smear-positive $\mathrm{TB}$ and the low probability that the majority of household contacts were exposed to viral infections within a short period prior to blood sampling. Furthermore, analysis of functional protein pathways revealed patterns characteristic of TB (type I interferon signalling, neutrophil mediated and inflammation responses) [27] and was consistent with the evolution of transcriptome signatures in macaques followed from time of infection (up-regulation of inflammation and interferon pathways was seen during the early phases post infection) [8]. Although interferon pathways are also evident in viral infections, the overall pattern of pathway activation is distinct from that seen in patients with common respiratory viral infections such as respiratory syncytial virus and influenza virus, in which Notch signalling [41] and ubiquitination signaling pathways [42] respectively, are distinctive host responses.

Although we did not find a relationship between the exposure signature and time from exposure, isoniazid prophylaxis, or the presence of lung PET/MRI abnormalities, this may simply reflect the small sample size and imprecision of estimates. The transcriptome measurements were made at a single timepoint, whereas studies in macaques indicate a dynamic situation with progressive attenuation after about 2 months following exposure [8]. Time from exposure was derived from subjective estimate of the onset of symptoms by the index case, and exposure likely comprises a series of repeated exposures over a prolonged period within a household prior to diagnosis and treatment of the index case. Isoniazid effects on bacterial replication may not directly affect the magnitude of the host immune response if this evolves independently of the underlying bacterial insult [43]. The time course of development and resolution of structural/metabolic changes in the PET/MRI is also unknown and may not follow the same pattern as those of the immune or inflammatory processes.

The finding of a higher TB exposure risk score in IGRA positive contacts provides additional support for validity of our signature. The positive transcriptome score in ten participants who were IGRA negative may reflect clearance of infection by innate immune responses preventing sufficient $\mathrm{TB}$ antigen exposure to stimulate memory $\mathrm{T}$-cell responses (detected by IGRA). Alternatively, the $\mathrm{T}$-cell responses could be present but below the threshold of detection of IGRA; up to $20 \%$ of people with active TB have a negative IGRA, [44] and the test has substantial variability, even for LTBI [45, 46]. It appears that our transcriptome signature is more sensitive than IGRA for detecting exposure, consistent with the finding of expression of a 20-gene signature of active TB in $26 \%$ of persistently IGRA-negative TB contacts (followed for up to 12 months after exposure) [25].

A test for recent TB exposure may be of value for TB control programmes or epidemiological researchers seeking to monitor ongoing TB exposure in the community. 


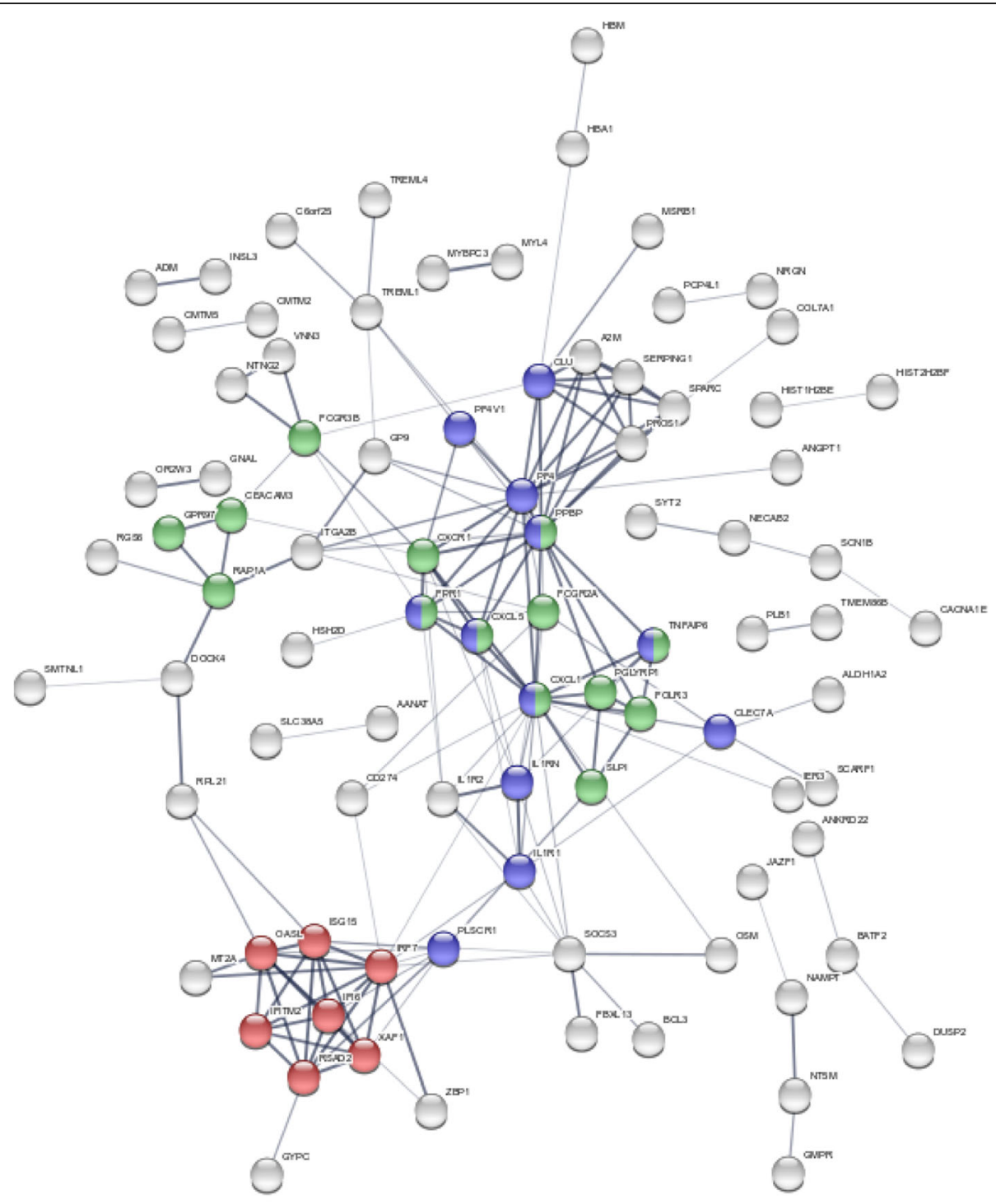

\section{GO Biological Process enrichment}

Type 1 interferon signaling pathway

\section{Inflammatory response}

Neutrophil mediated immunity

Fig. 1 Protein-protein network of the 186 exposure genes. Each circle represents a protein encoded by a gene. Colors were assigned proteins that belong to three overrepresented pathways relevant to TB infection. Type 1 interferon signaling pathway (red circles: IFI6, IFITM2, IRF7, ISG15, OASL, RSAD2, XAF1); Inflammatory response (blue circles: CLEC7A, CLU, CXCL1, CXCL5, FFAR2, FPR1, IL1R1, IL1RN, PF4, PF4V1, PLSCR1, PPBP, TNFAIP6, TPST10); Neutrophil mediated immunity (green circles: ANXA3, CEACAM3, CXCL1, CXCL5, CXCR1, FCGR2A, FCGR3B, FOLR3, FPR1, GPR97, PGLYRP1, PPBP, RAP1A, SLPI, TNFAIP6)

Traditional tests that require the administration of exogenous antigen at the time of the test (intra-dermal in the case of the TST or in vitro in the case of IGRA) measure durable immune responses that are largely independent of the timing of the original natural exposure [47]. Identifying recent natural (repeat) exposure is especially difficult in a high-burden setting where the background prevalence of IGRA or TST positivity is high. In contrast, the in vivo 
immune response to naturally-occurring infection measured by a transcriptome test should abate when infection is cleared and may therefore provide more precise temporal information of recent exposure, including repeat exposure in a high-burden setting. Such a test may be of value to identify environments and populations where there are high rates ('hot spots') of TB exposure and transmission and where active case-finding and infection control measures could be enhanced: more than half of prevalent culture positive TB disease is asymptomatic, and much transmission goes unrecognised $[8,9]$.

A transcriptome signature also has the potential to improve the selection of patients for preventive treatment. Traditional tests have a low positive predictive value for disease progression: IGRA/TST results did not add significantly to a clinical/demographic risk score, [48] and the excess risk associated with a positive versus negative IGRA/ TST is relatively modest [49]. Our study was not designed to identify a signature that would predict progression of active TB disease; however our signature contained many of the genes shown to predict disease progression in adolescents in South-Africa and contacts in the UK (two out of the three reported genes, BATF2 and SCARF1) [10, 36]. Our study lends further support to the potential of this approach for application in an Asian population.

Limitations of our study include the relatively small sample size, although it was comparable to other exploratory studies in the field and proved sufficient to identify a signature and analyse associated pathways. We did not collect information on socioeconomic status of contacts and controls that might partially confound the findings, although differences would be unlikely to account for the gene signature in the contacts, given that it included well known TB-associated genes and protein pathways. Our signature, although biologically plausible and consistent with published literature, would require validation in a large independent cohort prior to widespread use for the detection of recent TB exposure. We performed one transcriptome measurement per participant and did not examine evolution of the individual components of the signature over time. A macaque study (where the precise timing of exposure is known) found the greatest differential expression of genes from pre-infection values occurred between 20 to 56 days post-infection, and by day 120 following infection the whole blood signature had returned to baseline values [8]. The median time from estimated exposure to sampling in our study was 95 days which may have missed the peak response in some patients. However, a comprehensive and systematic description of longitudinal changes would require more precise knowledge of the timing of first exposure as well as collection of baseline samples soon after exposure, both of which are challenging.

\section{Conclusions}

We have expanded the scope of application of transcriptomics to identify a signature of recent TB exposure, independent of IGRA testing. Further validation studies and work to optimise the signature are needed, followed by validation of a reduced set of genes by RTPCR, but this research illustrates the potential for this approach to be applied for screening to identify areas of high TB exposure that could benefit from enhanced case finding and infection control measures; and supports the potential of transcriptomics to identify more precisely the individuals who would benefit from preventive treatment.

\section{Supplementary information}

Supplementary information accompanies this paper at https://doi.org/10. 1186/s12879-020-05116-1.

Additional file 1 Supplementary Figure 1. Panel A: Comparison of the 186 differentially expressed genes in contacts with 792 genes differentially expressed genes in patients with active TB (both compared to healthy controls) yielded 141 overlap genes. Panel B: Log fold change expression values of the 141 overlap genes for Contacts and active TB (Wilcoxon signed rank test $p<0.00001)$. Supplementary Table 1. Inclusion and exclusion criteria of household contact, active TB and healthy control groups. Supplementary Table 2. 186 genes from the comparison of exposed household contacts and healthy control (false discovery rate $<0.05$; log2fold change $>1$ or $<-1$ ). Supplementary Table 3. Genes in the exposure signature in this study that have been reported in other studies with active TB, latent and incipient TB. Numbers in cells are log2 fold change relative to comparison group (negative value represents downregulation); + , gene present but fold change not reported; -, gene not reported in study; ${ }^{*}$, IGRA positive vs IGRA-negative non-exposed controls; ${ }^{* *}$, IGRA/TST positive progressors vs nonprogressors. Supplementary Table 4. All overrepresented GO Biological Processes of the protein-protein network from STRING analysis. The three highlighted pathways are represented as colored circles in Fig. 1.

\section{Abbreviations}

TB: Tuberculosis; LTBI: Latent TB infection; TST: Tuberculin skin test; IGRA: Interferon gamma release assay; PET/MRI: Positron-emission tomography/Magnetic Resonance Imaging; PK/PD: Pharmacokinetics/ Pharmacodynamics

\section{Acknowledgements}

We thank Drs Kristina Rutkute, Pauline Yoong, and Meera Gurumurthy for administrative support; Pang Yan, Wang Jing and Lam Sek Onn for research nurse support; Professor TK Lim and colleagues in Division of Respiratory Medicine at NUH, and Drs Sonny Wang and Cynthia Chee at the Tuberculosis Control Unit, Singapore for assistance with demographic information; Collins W. Chu at the GIS for preliminary data analyses; Dr. John Totman and staff of the NUS Clinical Imaging Research Centre for performing the PET/MRI scans; Drs James Hallinan and Lih Kin Khor for clinical evaluation of the PET/MRI scans.

\section{Authors' contributions}

$\mathrm{PK}, \mathrm{BP}, \mathrm{PdS}, \mathrm{WL}, \mathrm{MH}, \mathrm{NP}$ designed the study; JM and $\mathrm{CN}$ recruited participants and collected the clinical data; PK, PdS, WL, ANMN performed sample analysis; PK and BP performed the bioinformatics analysis; all authors contributed to the interpretation of the data; NP wrote the first draft of the manuscript and all authors read and contributed to the final version.

\section{Funding}

This research is part of the Singapore Programme of Research Investigating New Approaches to Treatment of Tuberculosis (SPRINT-TB; www.sprinttb.org) 
and was supported by the Singapore Ministry of Health's National Medical Research Council under its TCR Flagship grant [NMRC/TCR/011-NUHS/2014]; a Clinician Scientist Award grant to N Paton [NMRC/CSA/0050/2013]; a Centre Grant supporting the National University of Singapore Yong Loo Lin School of Medicine BSL-3 Core Facility [NMRC/CG/013/2013]; by the National University of Singapore (start-up grant to N. Paton); and by the Singapore Agency for Science, Technology and Research (A*STAR) through core funding to the Genome Institute of Singapore. The funders had no role in study design, data collection and interpretation, or the decision to submit the work for publication.

\section{Availability of data and materials}

The datasets generated and/or analysed during the current study are available in NCBI Sequencing Read Archive under the BioProject accession PRJNA595691 (http://www.ncbi.nlm.nih.gov/595691).

\section{Ethics approval and consent to participate}

Ethics approval were approved by the Domain Specific Review Board of the National Healthcare Group, Singapore (NHG-DSRB; DSRB study codes 2012/ 00932, 2013/00116, and 2014/01269). All study participants provided written informed consent.

\section{Consent for publication}

Not applicable.

\section{Competing interests}

The authors report no conflict of interest.

\section{Author details}

${ }^{1}$ Department of Medicine, Yong Loo Lin School of Medicine, National University of Singapore, NUHS Tower Block Level 10, 1E Kent Ridge Road, Singapore 119228, Singapore. ${ }^{2}$ Genome Institute of Singapore, Agency for Science, Technology and Research, Singapore, Singapore. ${ }^{3}$ London School of Hygiene \& Tropical Medicine, London, UK. ${ }^{4}$ Department of Microbiology and Immunology, Yong Loo Lin School of Medicine, National University of Singapore, Singapore, Singapore.

\section{Received: 15 December 2019 Accepted: 24 May 2020} Published online: 09 June 2020

\section{References}

1. Getahun H, Matteelli A, Chaisson RE, Raviglione M. Latent mycobacterium tuberculosis infection. N Engl J Med. 2015;372(22):2127-35.

2. Cobelens F, Kik S, Esmail H, Cirillo DM, Lienhardt C, Matteelli A. From latent to patent: rethinking prediction of tuberculosis. Lancet Respir Med. 2017:5(4):243-4.

3. Houben RM, Dodd PJ. The global burden of latent tuberculosis infection: a reestimation using mathematical modelling. PLoS Med. 2016;13(10):e1002152.

4. Paton NI, Borand L, Benedicto J, Kyi MM, Mahmud AM, Norazmi MN, Sharma N, Chuchottaworn C, Huang Y-W, Kaswandani N. Diagnosis and management of latent tuberculosis infection in Asia: review of current status and challenges. Int J Infect Dis. 2019;87:21-9.

5. Yap P, Tan KHX, Lim WY, Barkham T, Tan LWL, Mark I, Chen C, Wang YT, Chee CBE. Prevalence of and risk factors associated with latent tuberculosis in Singapore: a cross-sectional survey. Int J Infect Dis. 2018;72:55-62.

6. Organization WH. The End TB Strategy. Global strategy and targets for tuberculosis prevention, care and control after 2015. Geneva: WHO; 2014. p. 2.

7. Salgame P, Geadas C, Collins L, Jones-López E, Ellner JJ. Latent tuberculosis infection-revisiting and revising concepts. Tuberculosis. 2015;95(4):373-84.

8. Gideon HP, Skinner JA, Baldwin N, Flynn JL, Lin PL. Early whole blood transcriptional signatures are associated with severity of lung inflammation in Cynomolgus macaques with mycobacterium tuberculosis infection. J Immunol. 2016;197(12):4817-28.

9. Dowdy DW, Basu S, Andrews JR. Is passive diagnosis enough? The impact of subclinical disease on diagnostic strategies for tuberculosis. Am J Respir Crit Care Med. 2013;187(5):543-51.

10. Zak DE, Penn-Nicholson A, Scriba TJ, Thompson E, Suliman S, Amon LM, Mahomed H, Erasmus M, Whatney W, Hussey GD, et al. A blood RNA signature for tuberculosis disease risk: a prospective cohort study. Lancet. 2016; 387(10035):2312-22.

11. Suliman S, Thompson EG, Sutherland J, Weiner J 3rd, Ota MO, Shankar S, Penn-Nicholson A, Thiel B, Erasmus M, Maertzdorf J. Four-gene pan-African blood signature predicts progression to tuberculosis. Am J Respir Crit Care Med. 2018;197(9):1198-208.

12. Drain PK, Bajema KL, Dowdy D, Dheda K, Naidoo K, Schumacher SG, Ma S, Meermeier E, Lewinsohn DM, Sherman DR. Incipient and subclinical tuberculosis: a clinical review of early stages and progression of infection. Clin Microbiol Rev. 2018;31(4):e00021-18.

13. Ghesani N, Patrawalla A, Lardizabal A, Salgame P, Fennelly KP. Increased cellular activity in thoracic lymph nodes in early human latent tuberculosis infection. Am J Respir Crit Care Med. 2014;189(6):748-50.

14. Molton JS, Thomas BA, Pang Y, Khor LK, Hallinan J, Naftalin CM, Totman J J Townsend DW, Lim TK, Chee CBE. Sub-clinical abnormalities detected by PET/MRI in household tuberculosis contacts. BMC Infect Dis. 2019;19(1):83.

15. Moosazadeh M, Khanjani N, Parsaee M. The prevalence of latent tuberculosis infection and smear positive pulmonary tuberculosis in people with household close contact with tuberculosis in north of Iran. Iran J Med Sci. 2015;40(2):161

16. Fox GJ, Barry SE, Britton WJ, Marks GB. Contact investigation for tuberculosis: a systematic review and meta-analysis. Eur Respir J. 2013;41(1):140-56.

17. Dobin A, Davis CA, Schlesinger F, Drenkow J, Zaleski C, Jha S, Batut P, Chaisson M, Gingeras TR. STAR: ultrafast universal RNA-seq aligner. Bioinformatics. 2013;29(1):15-21.

18. Anders S, Pyl PT, Huber W. HTSeq-a Python framework to work with highthroughput sequencing data. Bioinformatics. 2015;31(2):166-9.

19. Robinson MD, McCarthy DJ, Smyth GK. edgeR: a bioconductor package for differential expression analysis of digital gene expression data. Bioinformatics. 2010;26(1):139-40.

20. Benjamini $Y$, Hochberg Y. Controlling the false discovery rate: a practical and powerful approach to multiple testing. J R Stat Soc Ser B Methodol. 1995;57(1): 289-300.

21. Szklarczyk D, Franceschini A, Wyder S, Forslund K, Heller D, Huerta-Cepas J, Simonovic M, Roth A, Santos A, Tsafou KP. STRING v10: protein-protein interaction networks, integrated over the tree of life. Nucleic Acids Res. 2014;43(D1):D447-52.

22. Flannery BP, Teukolsky SA, Press WH, Vetterling WT. Numerical recipes in C: The art of scientific computing, vol. 2. Cambridge: Cambridge University Press; 1988.

23. Anderson ST, Kaforou M, Brent AJ, Wright VJ, Banwell CM, Chagaluka G, Crampin AC, Dockrell HM, French N, Hamilton MS. Diagnosis of childhood tuberculosis and host RNA expression in Africa. N Engl J Med. 2014;370(18):1712-23.

24. Kaforou M, Wright VJ, Oni T, French N, Anderson ST, Bangani N, Banwell CM, Brent AJ, Crampin AC, Dockrell HM. Detection of tuberculosis in HIVinfected and-uninfected African adults using whole blood RNA expression signatures: a case-control study. PLoS Med. 2013;10(10):e1001538.

25. Singhania A, Verma R, Graham CM, Lee J, Tran T, Richardson M, Lecine $P$, Leissner $\mathrm{P}$, Berry MP, Wilkinson RJ. A modular transcriptional signature identifies phenotypic heterogeneity of human tuberculosis infection. Nat Commun. 2018;9(1):2308.

26. Sing T, Sander $O$, Beerenwinkel N, Lengauer T. ROCR: visualizing classifier performance in R. Bioinformatics. 2005;21(20):3940-1.

27. Berry MP, Graham CM, McNab FW, Xu Z, Bloch SA, Oni T, Wilkinson KA, Banchereau R, Skinner J, Wilkinson RJ, et al. An interferon-inducible neutrophil-driven blood transcriptional signature in human tuberculosis. Nature. 2010;466(7309):973-7.

28. Bloom Cl, Graham CM, Berry MP, Wilkinson KA, Oni T, Rozakeas F, Xu Z, Rossello-Urgell J, Chaussabel D, Banchereau J. Detectable changes in the blood transcriptome are present after two weeks of antituberculosis therapy. PLoS One. 2012;7(10):e46191.

29. Jacobsen M, Repsilber D, Gutschmidt A, Neher A, Feldmann K, Mollenkopf HJ, Ziegler A, Kaufmann SH. Candidate biomarkers for discrimination between infection and disease caused by mycobacterium tuberculosis. J Mol Med. 2007;85(6):613-21.

30. Sambarey A, Devaprasad A, Mohan A, Ahmed A, Nayak S, Swaminathan S, D'Souza G, Jesuraj A, Dhar C, Babu S. Unbiased identification of blood-based biomarkers for pulmonary tuberculosis by modeling and mining molecular interaction networks. EBioMedicine. 2017;15:112-26.

31. Leong S, Zhao Y, Joseph NM, Hochberg NS, Sarkar S, Pleskunas J, Hom D, Lakshminarayanan S, Horsburgh CR Jr, Roy G. Existing blood transcriptional classifiers accurately discriminate active tuberculosis from latent infection in individuals from South India. Tuberculosis. 2018;109:41-51.

32. Ottenhoff $T H$, Dass RH, Yang N, Zhang MM, Wong HE, Sahiratmadja E, Khor CC, Alisjahbana B, van Crevel R, Marzuki S, et al. Genome-wide expression 
profiling identifies type 1 interferon response pathways in active tuberculosis. PLoS One. 2012;7(9):e45839.

33. Lee S-W, Wu LS-H, Huang G-M, Huang K-Y, Lee T-Y, Weng JT-Y. Gene expression profiling identifies candidate biomarkers for active and latent tuberculosis. In: BMC bioinformatics: 2016: BioMed Central; 2016. p. S3.

34. Bah SY, Forster T, Dickinson P, Kampmann B, Ghazal P. Meta-analysis identification of highly robust and differential immune-metabolic signatures of systemic host response to acute and latent tuberculosis in children and adults. Front Genet. 2018;9:457.

35. Maertzdorf J, Ota M, Repsilber D, Mollenkopf HJ, Weiner J, Hill PC, Kaufmann $\mathrm{SH}$. Functional correlations of pathogenesis-driven gene expression signatures in tuberculosis. PLoS One. 2011;6(10):e26938.

36. Roe J, Venturini C, Gupta R, Gurry C, Chain BM, Sun Y, Southern J, Jackson C, Lipman M, Miller RF. Blood transcriptomic stratification of short-term risk in contacts of tuberculosis. Clin Infect Dis. 2019:70(5):731-37.

37. Verhagen LM, Zomer A, Maes M, Villalba JA, del Nogal B, Eleveld M, van Hijum SA, de Waard JH, Hermans PW. A predictive signature gene set for discriminating active from latent tuberculosis in Warao Amerindian children. BMC Genomics. 2013;14(1):1.

38. Bloom Cl, Graham CM, Berry MP, Rozakeas F, Redford PS, Wang Y, Xu Z, Wilkinson KA, Wilkinson RJ, Kendrick Y. Transcriptional blood signatures distinguish pulmonary tuberculosis, pulmonary sarcoidosis, pneumonias and lung cancers. PLoS One. 2013;8(8):e70630

39. Lesho E, Forestiero FJ, Hirata MH, Hirata RD, Cecon L, Melo FF, Paik SH, Murata Y, Ferguson EW, Wang Z. Transcriptional responses of host peripheral blood cells to tuberculosis infection. Tuberculosis. 2011:91(5):390-9.

40. Mistry R, Cliff JM, Clayton CL, Beyers N, Mohamed YS, Wilson PA, Dockrell HM, Wallace DM, van Helden PD, Duncan K. Gene-expression patterns in whole blood identify subjects at risk for recurrent tuberculosis. J Infect Dis. 2007;195(3):357-65.

41. Do LAH, Pellet J, van Doorn HR, Tran AT, Nguyen BH, Tran TTL, Tran QH, Vo QB, Tran Dac NA, Trinh HN. Host transcription profile in nasal epithelium and whole blood of hospitalized children under 2 years of age with respiratory syncytial virus infection. J Infect Dis. 2017;217(1):134-46.

42. Hoang LT, Tolfvenstam T, Ooi EE, Khor CC, Naim ANM, Ho EXP, Ong SH, Wertheim HF, Fox A, Nguyen CW. Patient-based transcriptome-wide analysis identify interferon and ubiquination pathways as potential predictors of influenza a disease severity. PLoS One. 2014;9(11):e111640.

43. Biraro IA, Egesa M, Kimuda S, Smith SG, Toulza F, Levin J, Joloba M, Katamba A, Cose S, Dockrell HM. Effect of isoniazid preventive therapy on immune responses to mycobacterium tuberculosis: an open label randomised, controlled, exploratory study. BMC Infect Dis. 2015;15(1):438,

44. De Visser V, Sotgiu G, Lange C, Aabye MG, Bakker M, Bartalesi F, Brat K, Chee $\mathrm{CB}$, Dheda K, Dominguez J. False-negative interferon- $\gamma$ release assay results in active tuberculosis: a TBNET study. Eur Respir J. 2015;45(1):279-83.

45. Pai M, Denkinger CM, Kik SV, Rangaka MX, Zwerling A, Oxlade O, Metcalfe JZ, Cattamanchi A, Dowdy DW, Dheda K. Gamma interferon release assays for detection of mycobacterium tuberculosis infection. Clin Microbiol Rev. 2014;27(1):3-20.

46. Diel R, Goletti D, Ferrara G, Bothamley G, Cirillo D, Kampmann B, Lange C, Losi M, Markova R, Migliori G. Interferon- $\gamma$ release assays for the diagnosis of latent mycobacterium tuberculosis infection: a systematic review and metaanalysis. Eur Respir J. 2011;37(1):88-99.

47. Singhania A, Wilkinson RJ, Rodrigue M, Haldar P, O'Garra A. The value of transcriptomics in advancing knowledge of the immune response and diagnosis in tuberculosis. Nat Immunol. 2018;19(11):1159.

48. Saunders MJ, Wingfield T, Tovar MA, Baldwin MR, Datta S, Zevallos K, Montoya R, Valencia TR, Friedland JS, Moulton LH. A score to predict and stratify risk of tuberculosis in adult contacts of tuberculosis index cases: a prospective derivation and external validation cohort study. Lancet Infect Dis. 2017;17(11):1190-9.

49. Rangaka MX, Wilkinson KA, Glynn JR, Ling D, Menzies D, MwansaKambafwile J, Fielding K, Wilkinson RJ, Pai M. Predictive value of interferon- $\gamma$ release assays for incident active tuberculosis: a systematic review and meta-analysis. Lancet Infect Dis. 2012;12(1):45-55.

\section{Publisher's Note}

Springer Nature remains neutral with regard to jurisdictional claims in published maps and institutional affiliations.

Ready to submit your research? Choose BMC and benefit from:

- fast, convenient online submission

- thorough peer review by experienced researchers in your field

- rapid publication on acceptance

- support for research data, including large and complex data types

- gold Open Access which fosters wider collaboration and increased citations

- maximum visibility for your research: over $100 \mathrm{M}$ website views per year

At BMC, research is always in progress.

Learn more biomedcentral.com/submissions 